\title{
Apoptosis inhibitor of macrophage (AIM) expression in alveolar macrophages in COPD
}

\author{
Jun Kojima', Jun Araya ${ }^{1 *}$, Hiromichi Hara', Saburo Ito ${ }^{1}$, Naoki Takasaka ${ }^{1}$, Kenji Kobayashi', Satoko Fujii', \\ Chikako Tsurushige ${ }^{1}$, Takanori Numata ${ }^{1}$, Takeo Ishikawa ${ }^{1}$, Kenichiro Shimizu ${ }^{1}$, Makoto Kawaishi $^{1}, K^{2}$ Keisuke Saito ${ }^{1}$, \\ Noriki Kamiya ${ }^{2}$, Jun Hirano ${ }^{2}$, Makoto Odaka², Toshiaki Morikawa² ${ }^{2}$ Hiroshi Hano ${ }^{3}$, Satoko Arai ${ }^{4}$, Toru Miyazaki ${ }^{4}$, \\ Yumi Kaneko', Katsutoshi Nakayama' and Kazuyoshi Kuwano'
}

\begin{abstract}
Background: Marked accumulation of alveolar macrophages (AM) conferred by apoptosis resistance has been implicated in pathogenesis of chronic obstructive pulmonary disease (COPD). Apoptosis inhibitor of macrophage (AIM), has been shown to be produced by mature tissue macrophages and AIM demonstrates anti-apoptotic property against multiple apoptosis-inducing stimuli. Accordingly, we attempt to determine if AIM is expressed in $\mathrm{AM}$ and whether AIM is involved in the regulation of apoptosis in the setting of cigarette smoke extract (CSE) exposure.

Methods: Immunohistochemical evaluations of AIM were performed. Immunostaining was assessed by counting total and positively staining AM numbers in each case ( $n=5$ in control, $n=5$ in non-COPD smoker, $n=5$ in COPD). AM were isolated from bronchoalveolar lavage fluid (BALF). The changes of AIM expression levels in response to CSE exposure in AM were evaluated. Knock-down of anti-apoptotic BCl-xL was mediated by siRNA transfection. U937 monocyte-macrophage cell line was used to explore the anti-apoptotic properties of AIM.

Results: The numbers of AM and AIM-positive AM were significantly increased in COPD lungs. AIM expression was demonstrated at both mRNA and protein levels in isolated AM, which was enhanced in response to CSE exposure. AIM significantly increased $B C l-x L$ expression levels in $A M$ and $B C l-x L$ was involved in a part of anti-apoptotic mechanisms of AIM in U937 cells in the setting of CSE exposure.
\end{abstract}

Conclusions: These results suggest that AIM expression in association with cigarette smoking may be involved in accumulation of AM in COPD.

Keywords: AIM, Alveolar macrophage, Apoptosis, COPD

\section{Background}

Chronic obstructive pulmonary disease (COPD) is one of the leading causes of death worldwide and chronic cigarette smoke is sufficient to trigger COPD development [1]. Alveolar macrophages (AM) orchestrate innate immune responses for host defense through pattern recognition receptors (PRRs), thus marked increase of AM in response to cigarette smoke exposure has been widely implicated in pathogenesis of COPD via excessive

\footnotetext{
* Correspondence: md986001@yahoo.co.jp

'Department of Internal Medicine, Division of Respiratory Diseases, Jikei University School of Medicine, 3-25-8 Nishi-shimbashi, Minato-ku, Tokyo 105-8461, Japan

Full list of author information is available at the end of the article
}

inflammatory cytokine, chemokine, and protease secretions, resulting in lung tissue destruction and airway remodeling [2-5]. In addition to an inverse correlation between the number of AM and airflow limitation in COPD [6], reduction of AM by neutralization of granulocyte/macrophage colony-stimulating factor (GMCSF) attenuated cigarette smoke-induced lung inflammation in mouse models [7]. Therefore, to elucidate the mechanism of AM accumulation may offer potential clues to understand COPD pathogenesis. However it remains unclear whether the increase in number of AM in COPD lung results from increased flux from circulating monocytes, local AM proliferation, or prolonged AM survival conferred by apoptosis resistance $[2,3]$.

\section{Biomed Central}

(c) 2013 Kojima et al.; licensee BioMed Central Ltd. This is an Open Access article distributed under the terms of the Creative Commons Attribution License (http://creativecommons.org/licenses/by/2.0), which permits unrestricted use, distribution, and reproduction in any medium, provided the original work is properly cited. 
Apoptosis, a type of programmed cell death, is a physiologic mechanism for cell deletion without inflammation, which is necessary for the maintenance of homeostatic plasticity in the lung [8]. However, the cell type specific imbalance of positive and negative regulation of apoptosis has been proposed to be a critical determination of disease progression in COPD. For instance, excessive apoptotic cell loss of the alveolar epithelial cells and endothelial cells has been postulated to be a potential cause for development of lung tissue destruction, emphysema [9]. Conversely, reduced apoptosis through a lack of pro-apoptotic p53 expression [10] and an increase in anti-apoptotic $\mathrm{Bcl}-\mathrm{xL}$ and the cytoplasmic form of $\mathrm{p} 21^{\mathrm{CIP} 1 / \mathrm{WAF} 1}$ has been reported in AM from smokers in association with chronicity of inflammation in COPD pathogenesis [11]. However the AM specific mechanism of anti-apoptotic property for prolonged cell survival in COPD lung has not been clearly elucidated.

Apoptosis inhibitor of macrophage (AIM), a member of scavenger receptor cysteine-rich superfamily, has been shown to be exclusively produced by mature tissue macrophages [12]. In mouse models, AIM contributes to the development of atherosclerotic lesions by conferring apoptosis resistance to cytotoxic oxidized-low density lipoprotein (oxLDL) in macrophages [13]. AIM is also involved in apoptosis regulation for $\mathrm{T}$ cell and natural killer $\mathrm{T}$ (NKT) cells in the model of corynebacteriuminduced granuloma formation [14]. Furthermore, the anti-apoptotic property of AIM has been demonstrated against multiple apoptosis-inducing stimuli, including dexamethasone, irradiation, and Fas/CD95 [12]. Hence, we speculate the potential involvement of AIM in prolonged survival for AM under stress conditions of cigarette smoke exposure, which can be a part of mechanism for accumulation of AM in pathogenic sequence for COPD development. However both AIM expression in human lung and involvement of AIM in regulation of apoptosis in the setting of cigarette smoke exposure remains to be determined.

In this context, we examined the expression of AIM in lung tissues from COPD patients by means of immunohistochemial evaluation. We also examined the expression of AIM in AM isolated from bronchoalveolar lavage fluid (BALF) and also evaluated the changes of expression levels of AIM in response to cigarette smoke extract (CSE) exposure. Anti-apoptotic properties of AIM in CSE-induced apoptosis were also explored in in vitro models.

\section{Methods}

\section{Immunohistochemical examinations}

Lung tissue samples for immunohistochemistry were collected from pneumonectomy and lobectomy specimens from resections performed for primary lung cancer and tissue cancer involvement was excluded by histological examinations. Informed consent was obtained from all surgical participants as part of an approved ongoing research protocol by the ethical committee of Jikei University School of Medicine. Immunohistochemistry was performed as previously described with minor modification on the paraffin-embedded lung tissues [15]. The anti-AIM antibody SA-1, available for both mouse and human AIM recognition, was produced by immunizing rabbits with recombinant mouse AIM [12]. AM number and AIM staining were assessed by counting total and positively staining cells in five randomly selected lung fields at a magnification of $\times 400$ in each case. Counting was only performed for cells in the airspace, which were morphologically recognized as alveolar macrophages. ( $\mathrm{n}=5$ in control, $\mathrm{n}=5$ in non-COPD smoker, $\mathrm{n}=5$ in COPD). Patient characteristic features are presented in Table 1.

\section{Cell culture and reagents}

AM were isolated from bronchoalveolar lavage fluid (BALF) obtained from non-COPD patients. Filtered BALF was centrifuged at $400 \mathrm{~g}$ for $10 \mathrm{~min}$. The cells were allowed to adhere to culture dishes in RPMI1640 with $10 \% \mathrm{FCS}$ at $37^{\circ} \mathrm{C}$ for $2 \mathrm{~h}$ and nonadherent cells were removed. The adherent cells were microscopically confirmed as AM (>90\%) [16]. U937, purchased from American type culture collection, were grown in RPMI1640 with $10 \%$ fetal bovine serum and penicillinstreptomycin.

Antibodies used were goat anti-AIM (AnaSpec, San Jose, CA), rabbit anti-Bcl-2 (Cell signaling Technology, Tokyo, Japan), rabbit anti-Bcl-xL (Cell signaling Technology, Tokyo, Japan), rabbit anti-caspase-8 (Cell signaling Technology, Tokyo, Japan), rabbit anti-caspase-9 (Cell signaling Technology, Tokyo, Japan), and mouse anti- $\beta$-actin (Santa Cruz, Santa Cruz, CA).

\section{Preparation of cigarette smoke extract}

Cigarette smoke extract (CSE) was prepared as previously described [17]. Forty milliliters of cigarette smoke was drawn into the syringe and slowly bubbled into sterile serum-free cell culture media in 15-ml BD falcon tube. One cigarette was used for the preparation of 10 milliliters of solution. CSE solution was filtered $(0.22 \mu \mathrm{m})$ to remove insoluble particles and was designated as a 100\% CSE solution.

\section{Western blotting}

AM and U937 cells grown on 6-well culture plates were lysed in 1X SDS sample buffer or RIPA buffer (Thermo Fisher Scientific, Waltham, MA) with protease inhibitor cocktail (Roche Diagnostics, Tokyo, Japan) and $1 \mathrm{mM}$ sodium orthovanadate (Sigma Aldrich, Tokyo, Japan). 
Table 1 Patient characteristics

\begin{tabular}{|c|c|c|c|c|}
\hline & $\begin{array}{c}\text { Non-smoker } \\
(n=5)\end{array}$ & $\begin{array}{l}\text { Non-COPD smoker } \\
\qquad(n=5)\end{array}$ & $\begin{array}{l}\text { COPD } \\
(n=5)\end{array}$ & $p$ value \\
\hline Age, years & $62.6 \pm 16.7$ & $62.0 \pm 12.1$ & $63.6 \pm 3.5$ & NS \\
\hline Male, $\%$ of group & 40 & 80 & 100 & NA \\
\hline $\mathrm{SI}$ & 0 & $38.3 \pm 21.8^{*}$ & $78.5 \pm 68.7$ & ${ }^{*} p<0.05$ \\
\hline FEV1/FVC & $79.0 \pm 6.5$ & $76.7 \pm 2.9$ & $57.9 \pm 13.5 *$ & ${ }^{*} p<0.05$ \\
\hline$\%$ VC & $109.1 \pm 19.8$ & $104.4 \pm 16.55$ & $97.3 \pm 18.7$ & NS \\
\hline
\end{tabular}

Definition of abbreviations: COPD = chronic obstructive pulmonary disease, FEV1 = forced expiratory volume in 1 second, $\mathrm{SI}=\mathrm{Smoking}$ Index (pack/ year), VC = vital capacity, NA = not assessed, NS = not statistically significant. Values are mean \pm SD.

Western blotting was performed as previously described with minor modification [15]. After transfer to PVDF membrane (Immobilon-P, Millipore, MA), blotting with specific primary antibodies were performed overnight at $4^{\circ} \mathrm{C}$. Proteins were detected by HRP-conjugated secondary antibody (Cell signaling Technology, Tokyo, Japan) followed by chemiluminescence detection (ECL; GE Healthcare,Tokyo, Japan) with a LAS-4000 UV mini system (Fujifilm, Shiga, Japan).

\section{Plasmids, siRNA, and transfection}

The AIM expression vector was generated by inserting humanAIM cDNA into the pCAGGS vector cassette. The BcL-xL and negative control siRNAs were purchased (Applied Biosystems Life Technologies Japan, Tokyo, Japan) and transfections of AM and U937 were performed using the Neon ${ }^{\circledR}$ Transfection System (Invitrogen Life Technologies Japan, Tokyo, Japan), using matched optimized transfection kits.

\section{Preparation of conditioned medium containing AIM}

Human Embryonic Kidney (HEK) 293 cells were transfected with control or AIM expression vector by using TransIT ${ }^{\circledR}$-LT1 Transfection Reagent (Mirus Bio LLC, WI) according to the manufacturer's instruction. Conditioned medium without FCS preparation was started $24 \mathrm{~h}$ post-transfection and was collected after $48 \mathrm{~h}$ incubation. Collected conditioned medium and fresh RPMI1640 without FCS were mixed equally for further experiments.

\section{RNA isolation, quantitative polymerase chain reaction}

RNA isolation, reverse transcription and Real-Time PCR were performed using the SYBR green method as previously described [15]. The primers used were AIM sense primer, 5'- TTCTCCTTGATCCTTGCCATTTG -3'; AIM antisense primer, 5'- ACTGGCCTTTCTGTTCCACC -3'; $\beta$-actin sense primer 5 -CATGTACGTTGCTATCCAGG C-3' $\beta$-actin antisense primer 5'-CTCCTTAATGTCAC GCACGAT-3'. These primer sets yielded PCR products of $121 \mathrm{bp}$ and $250 \mathrm{bp}$ for AIM and $\beta$-actin respectively.
Primer sequences for $\beta$-actin were from Primer Bank (http://pga.mgh.harvard.edu/primerbank).

\section{Detection of apoptosis and immunofluorescence staining}

Cell cycle analysis and flow cytometry were performed as previously described [18]. Briefly, treated cells were harvested and immediately immobilized by $70 \%$ ice-cold ethanol overnight. Then the cells were incubated with $100 \mu \mathrm{g} / \mathrm{ml}$ of RNase and $50 \mu \mathrm{g} / \mathrm{ml}$ of propidium iodide (PI) (Sigma Aldrich, Tokyo, Japan) in PBS-Triton X-100 $(0.05 \%)$ for $40 \mathrm{~min}$ at $37^{\circ} \mathrm{C}$. The quantity of cells with hypodiploid DNA was measured on a FACScan at the FL-2 channel (Becton Dickinson). The percentage of control cells with hypodiploid DNA was designated as $100 \%$ cell death in each experiment.

DNA fragmentation analysis was performed as previously described [18]. After treatment, collected cells were lysed in $100 \mu \mathrm{l}$ of cell lysis buffer $(10 \mathrm{mM}$ Tris $\bullet \mathrm{HCl}$, pH7.4, 10 mM EDTA, pH8.0, and 0.5\% TritonX-100). After centrifugation, the supernatant was treated with $2 \mu \mathrm{l}$ of RNase A $(20 \mathrm{mg} / \mathrm{ml})$ for $30 \mathrm{~min}$ at $37^{\circ} \mathrm{C}$ and then incubated with $2 \mu$ lof proteinase $\mathrm{K}(20 \mathrm{mg} / \mathrm{ml})$ for $30 \mathrm{~min}$ at $37^{\circ} \mathrm{C}$. DNA in the supernatant was precipitated overnight by the addition of $20 \mu \mathrm{l} 5 \mathrm{M} \mathrm{NaCl}$ and $120 \mu \mathrm{l}$ isopropanol. After centrifugation, the DNA pellet was dissolved in Tris-EDTA buffer followed by electrophoresis on a $2 \%$ agarose gel. The agarose gel was stained with ethidium bromide, and the resulting DNA fragmentation pattern was revealed by ultraviolet illumination.

Fluorescence microscopic detection of apoptotic cells was performed as previously described [18]. Harvested cells were stained with 2'-(4-Hydroxyphenyl)-5-(4-methyl1-piperazinyl)-2,5'-bi-1H-benzimidazole (Hoechst 33258: Sigma Aldrich, Japan), and then seeded on a glass slide and photographed with a fluorescent microscope. Percentage of apoptotic cells was assessed by manual counting, three hundred cells per condition.

\section{Statistics}

Data are shown as the average $( \pm$ SEM $)$ taken from at least three independent experiments. Student's t-test was used for comparison of two data sets. Comparison 


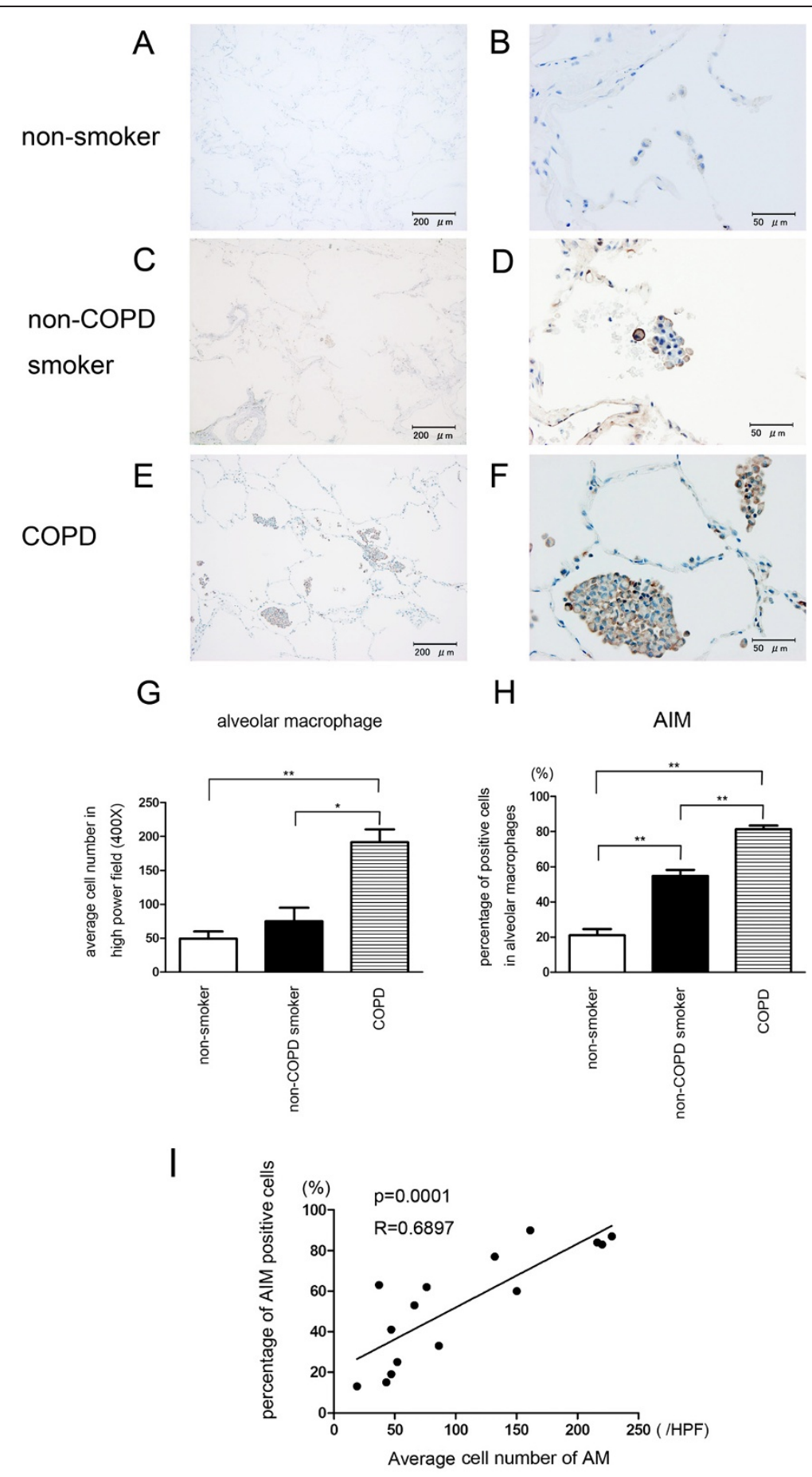

Figure 1 AIM expression in alveolar macrophages in lung tissues. Immunohistochemical staining of AIM in AM in lung tissues from nonsmoker, non-COPD smoker, and COPD patients: Photomicrographs of non-smoker lung (A and $\mathbf{B})$, non-COPD smoker lung (C and D), and COPD lung tissues $(\mathbf{E}$ and $\mathbf{F})$. (G) Shown is the average total number of AM ( \pm SEM) in five randomly selected lung fields in high power field (HPF) (X400) from five cases. Open bar is non-smoker, filled bar is non-COPD smoker, and horizontal crosshatched bar is COPD. ${ }^{*} p<0.05,{ }^{* *} p<0.001$. (H) Shown is the average \pm SEM of percentages of AIM positive cells in total cells in HPF (X400) from five cases. Open bar is non-smoker, filled bar is non-COPD smoker, and horizontal crosshatched bar is COPD. ${ }^{* *} \mathrm{p}<0.001$. (I) Shown is the relationship between number of AM and percentages of AlM positive cells. Bar $=200 \mu \mathrm{m}$ in $\mathbf{A}, \mathbf{C}$, and $\mathbf{E}$. Bar $=50 \mu \mathrm{m}$ in $\mathbf{B}, \mathbf{D}$, and $\mathbf{F}$. 
between groups was made using one-way ANOVA followed by the Tukey test. Linear regression analysis was used to compare average cell number of AM in high power field (X400) to percentage of AIM positive AM. Significance was defined as $\mathrm{p}<0.05$. Statistical software was Prism v.5 (GraphPad Software, Inc., San Diego, CA).

\section{Results}

\section{AM accumulation and AIM expression in COPD lung}

The number of AM was significantly increased in the lungs of COPD patients but not significant in non-COPD smoker compared to non-smoker (Figure $1 \mathrm{~A}$ to $\mathrm{G}$ ). AIM expression was clearly observed in AM in lung of COPD patients and the percentages of positively staining AM were significantly increased in both COPD patients and non-COPD smoker compared to nonsmoker (Figure 1F, H). AIM staining was also lightly present in alveolar walls in lung tissues from smokers.
Interestingly, the number of AM correlated significantly with increasing percentage of AIM positive AM, indicating the potential link between increasing AM accumulation and AIM expression (Figure 1I).

\section{CSE induces AIM expression in AM}

We next examined the expression of AIM in AM isolated from BALF. AIM expression was demonstrated in AM and CSE significantly increased AIM expression at both mRNA and protein levels in AM and maximum effect was observed with 1\% CSE (Figure 2A, C). However, significant induction was not observed with 5\% CSE, which might be attributed to cytotoxic effect of higher concentration of CSE. CSE (1\%) induced AIM expression peaked at $16 \mathrm{~h}$ incubation at both mRNA and protein levels (Figure 2B, D). Protein level in cell lysate was decreased after $32 \mathrm{~h}$ incubation, which may reflect the secretory nature of AIM.
A

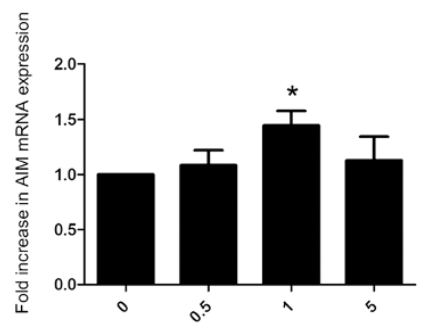

(CSE \%)

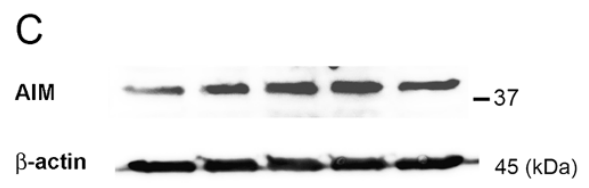

(CSE \%)

B

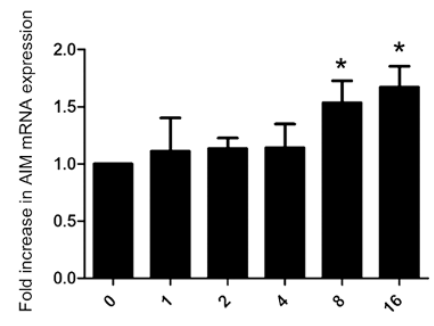

(hour)
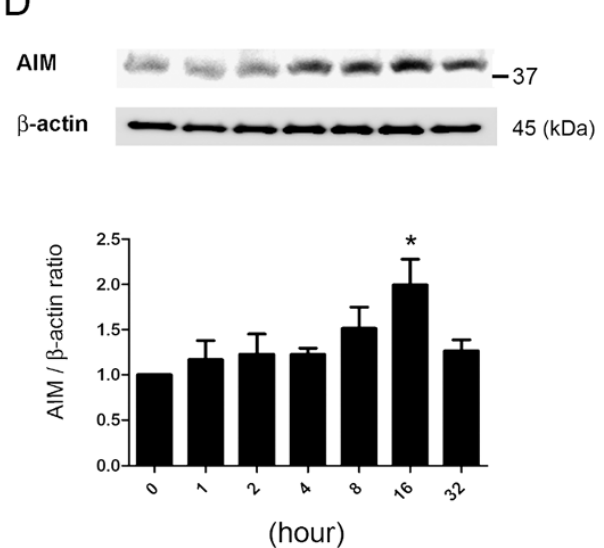

Figure 2 AIM expression in alveolar macrophages isolated from bronchoalveolar lavage fluid. (A) AM were treated with indicated concentrations of cigarette smoke extract (CSE) for $16 \mathrm{~h}(n=5)$. Real time-PCR was performed using primers to AIM or $\beta$-actin, as a control. AIM expression was normalized to $\beta$-actin. Shown is the fold increase $\left( \pm\right.$ SEM) relative to control treated cells. ${ }^{*} p<0.05$. (B) AM were treated with CSE $(1.0 \%)$ and RNA were harvested at indicated time points $(n=4)$. Real time-PCR was performed using primers to AIM or $\beta$-actin. AlM expression was normalized to $\beta$-actin. Shown is the fold increase ( \pm SEM) relative to control cells. ${ }^{*} p<0.05$. (C) Western blotting (WB) using anti-AlM and anti$\beta$-actin of cell lysates from indicated concentrations of CSE treated AM (upper panel). Cell lysates were collected after $16 \mathrm{~h}$ treatment. Shown is a representative experiment of 3 showing similar results. AIM was normalized to $\beta$-actin. The lower panel is the average ( \pm SEM) of relative changes to control cells. (D) WB using anti-AIM and anti- $\beta$-actin of cell lysates from CSE (1.0\%) treated AM (upper panel). Cell lysates were collected at indicated time points. Shown is a representative experiment of 3 showing similar results. AIM was normalized to $\beta$-actin. The lower panel is the average $( \pm$ SEM) of relative changes to control cells. 


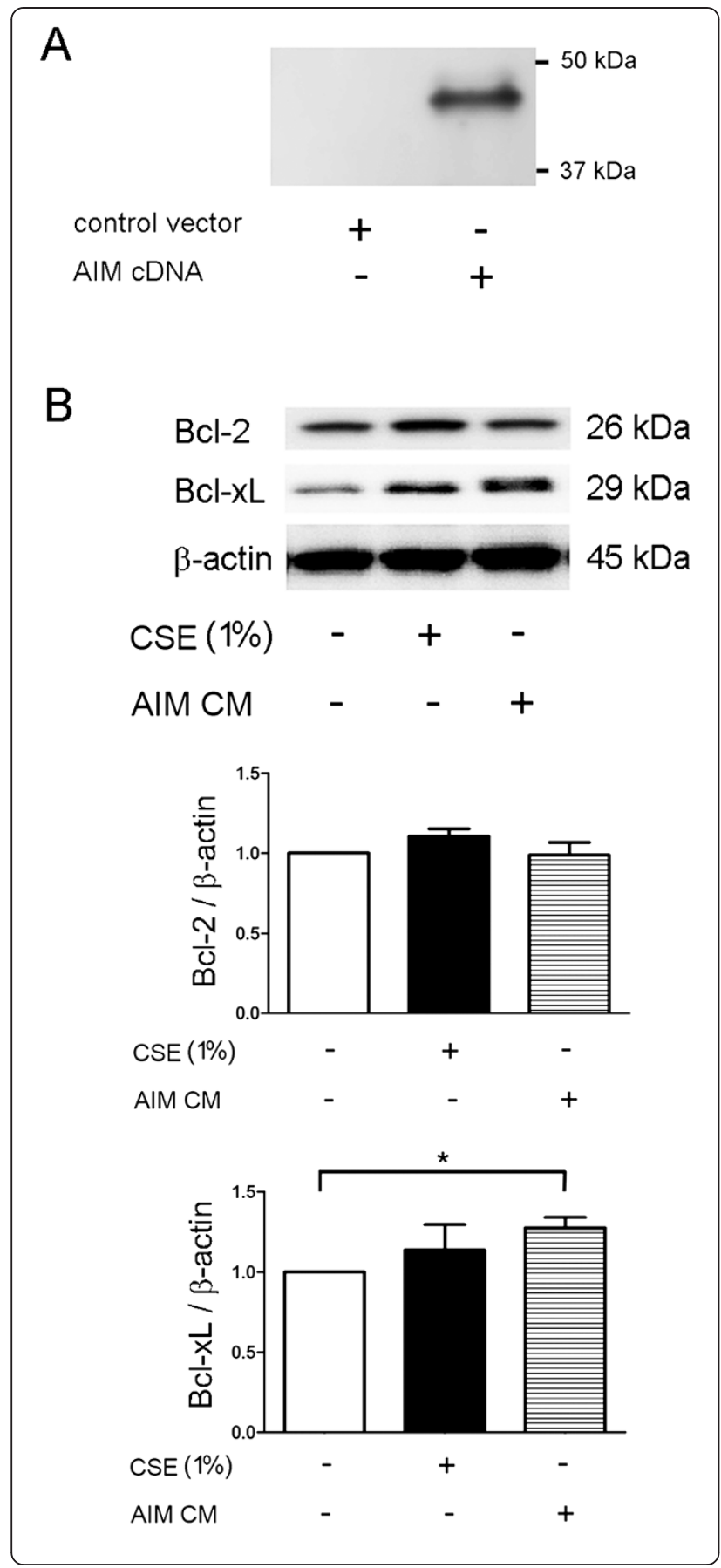

Figure 3 The changes of $\mathrm{Bcl}-2$ and $\mathrm{Bcl}-\mathrm{xL}$ expression levels by AIM in alveolar macrophages. (A) Western blotting (WB) using anti-AIM of conditioned medium from control empty vector (lane 1) or AIM expression vector (lane 2) transfected HEK293 cells. Conditioned medium was collected after $48 \mathrm{~h}$ incubation. (B) WB using anti-BCl-2, anti-BCl-xL, and anti- $\beta$-actin of cell lysates of alveolar macrophages (AM) treated with control conditioned medium (lane 1), control conditioned medium containing CSE (1.0\%) (lane 2), and conditioned medium containing AIM (lane 3)(upper panel). Cell lysates of AM were collected after $24 \mathrm{~h}$ treatment. Shown is a representative experiment of 3 showing similar results. The middle panel is the average ( \pm SEM) of relative changes in $\mathrm{BCl}-2$ normalized to $\beta$-actin. The lower panel is the average $( \pm$ SEM) of relative changes in $\mathrm{BCl}-\mathrm{xL}$ normalized to $\beta$-actin. Open bar is control conditioned medium treated, filled bar is control conditioned medium treated in the presence of CSE (1.0\%), and horizontal crosshatched bar is conditioned containing AIM treated. ${ }^{*} p<0.05$.

\section{AIM increases $\mathrm{Bcl}-\mathrm{xL}$ expression levels in $\mathrm{AM}$}

$\mathrm{Bcl}-\mathrm{xL}$ has been implicated in prolonged survival for AM in COPD lung [11]. Hence, to clarify the AIMmediated anti-apoptotic mechanism in AM, antiapoptotic $\mathrm{Bcl}-2$ family proteins of $\mathrm{Bcl}-2$ and $\mathrm{Bcl}-\mathrm{xL}$ were evaluated. To prepare the conditioned medium containing recombinant AIM, HEK 293 cells were transfected with the AIM expression vector. Secretion of AIM in conditioned medium was confirmed by western blotting using anti-AIM antibody (Figure 3A). Intriguingly, we observed a significant increase in Bcl-xL expression levels in AM incubated with conditioned medium containing AIM (Figure 3B). However no significant increase was observed in the expression levels of Bcl-2, suggesting the specific induction of Bcl-xL by AIM in AM. Interestingly, non-significant increase of both Bcl-2 and Bcl-xL levels was also observed in response to CSE exposure.

\section{AIM inhibits CSE-induced apoptosis in U937cells}

To evaluate the inhibitory role of AIM in CSE-induced apoptosis, we employed U937 monocyte-macrophage cell line without AIM expression (data not shown). CSE (5.0\%) clearly induced apoptosis in U937 cells by means of nuclear staining with Hoechst 33258, DNA laddering, and FACS analysis for percentage of cells with hypodiploid DNA (Figure 4A to C).

CSE has been demonstrated to induce apoptosis through both the mitochondrial and death receptor pathways [19]. Therefore, to clarify which pathway is dominantly involved in CSE-induced apoptosis, we evaluated the caspase- 8 and -9 activation by detecting cleaved active form by western blotting. CSE apparently activated both caspase- 8 and caspase-9, indicating that both extrinsic and intrinsic apoptosis pathway are involved in CSEinduced apoptosis in U937 cell (Figure 4D). U937 cells cultured in the conditioned medium containing AIM were also treated with CSE. Interestingly, CSE-induced 
B
A
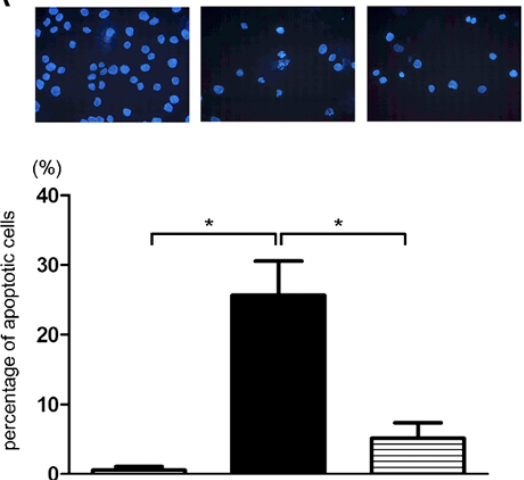

CSE

(5.0\%)

AIM CM - $\quad$ - $\quad+$

C

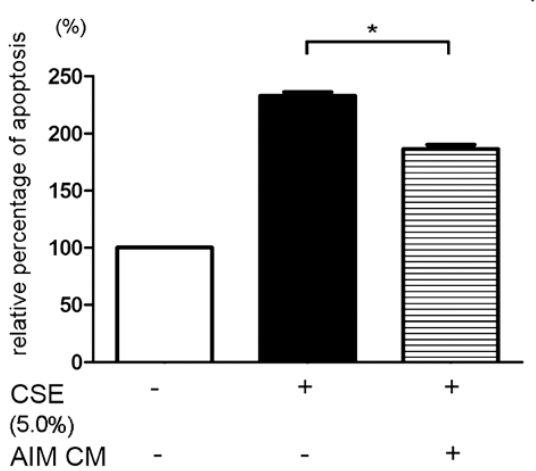

E
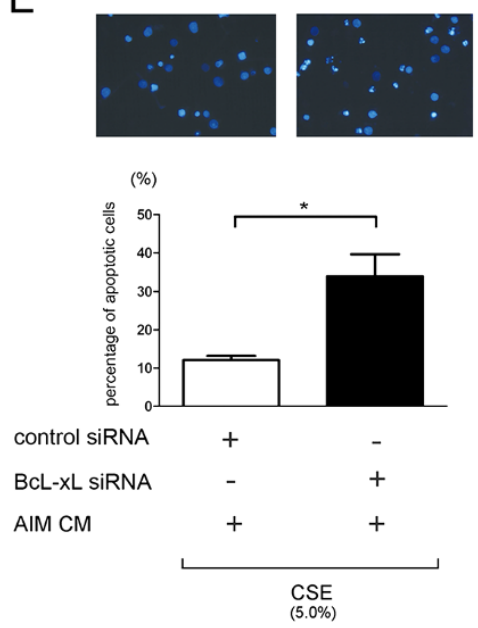

D

pro- caspase-8

$57 \mathrm{kDa}$

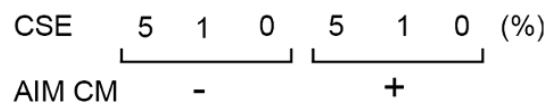

AIM CM - $\quad+$

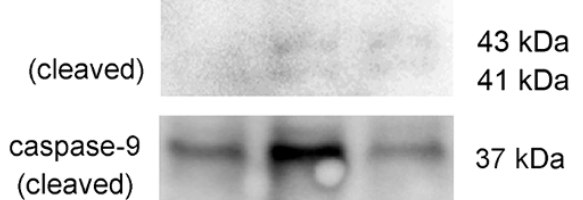

$\beta$-actin

$\rightarrow$

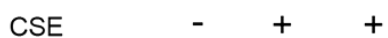

(5.0\%)

AIM CM

$\mathrm{F}$

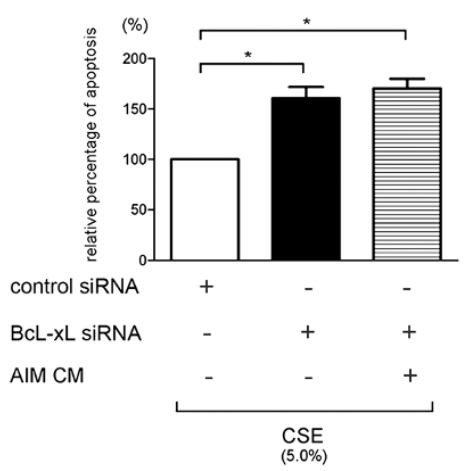




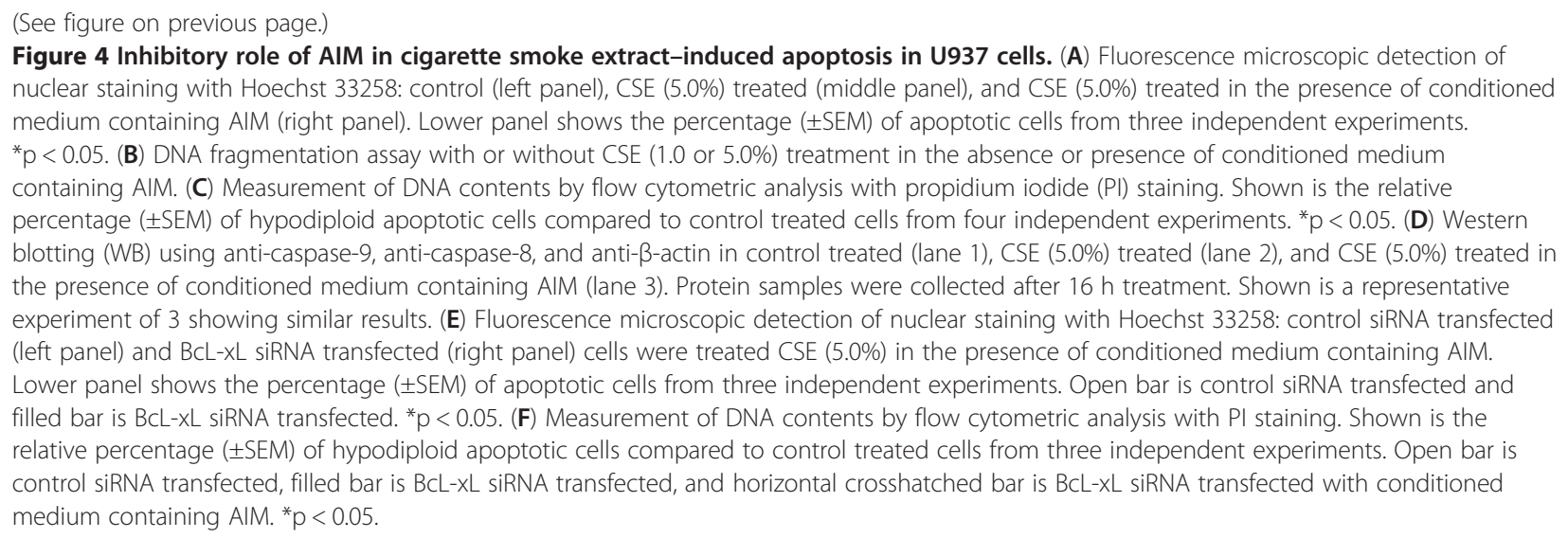

apoptosis was clearly suppressed by culturing in conditioned medium containing AIM. (Figure 4A to C). Interestingly, only caspase- 9 activation was inhibited in the presence of AIM. To confirm the involvement of Bcl-xL in the AIM-mediated anti-apoptotic mechanism, U937 cells were transfected with $\mathrm{Bcl}-\mathrm{xL}$ siRNA, resulting in decreased anti-apoptosis property of conditioned medium containing AIM (Figure 4E, F).

\section{Discussion}

Although a previous paper failed to show AIM expression in the lung of murine models, which can be attributed to relatively small amount of expression levels with methodological limitations or species specific expression pattern of AIM in AM, the present study elucidated that human AM express AIM [12]. We consider that the increase in AIM expression in response to CSE exposure is an important clue for understanding the role of AIM in human lung pathophysiology. Our immunohistochemistry clearly demonstrated AIM expression in AM, which appeared to be associated with increase in number of AM in COPD lung. Intriguingly, slight AIM staining was also observed in alveolar walls in lung tissues from smokers, which can be attributed to the secretory nature of AIM, suggesting a potential role for AIM in paracrine regulation of alveolar epithelial cells and endothelial cells, which has yet to be determined. We also demonstrated the anti-apoptotic nature of AIM in CSEinduced apoptosis in U937 monocyte-macrophage cell line, which was at least partly attributed to increased expression of an anti-apoptotic Bcl-2-family protein, Bcl-xL.

The anti-apoptotic nature of AIM has been demonstrated to be against to multiple apoptosis-inducing stimuli, including dexamethasone, irradiation, and Fas/ CD95, hence AIM may exert multiple mechanisms in anti-apoptotic regulation, but the detail remains to be determined [12]. Although the involvement of Bcl-2 and $\mathrm{Bcl}-\mathrm{x}$ has been excluded from AIM-mediated anti- apoptosis in thymocytes, we demonstrated significantly increased Bcl-xL expression in AM in response to AIM (Figure 3). Because of the apoptosis resistance of AM in in vitro culture conditions, we used U937 cells as an experimental model to clarify the anti-apoptotic mechanism of AIM. Indeed, no apparent increase in cell death of AM by CSE (5.0\%) exposure was observed by means of trypan blue dye exclusion (data not shown). Dominant inhibition of mitochondrial intrinsic apoptotic pathway of caspase-9 activation by AIM and inhibition of anti-apoptotic property of AIM by knockdown experiments, support the notion that $\mathrm{Bcl}-\mathrm{xL}$ is involved in a part of anti-apoptotic mechanisms of AIM in the setting of CSE exposure. Therefore, we speculate that AIM may at least partly account for the previous finding of increased $\mathrm{Bcl}-\mathrm{xL}$ expression in AM from smoker in association with apoptosis resistance in COPD lung [11]. However, we understand the potential limitations of using U937 to elucidate the anti-apoptotic mechanisms found in AM, hence more relevant in vitro and in vivo models are needed to further confirm the physiological importance of AIM in COPD pathology.

AM phenotype is largely divided into M1 and M2 polarization based on differences in patterns of cell surface receptor expression. The M1 polarization-induced by interferon- $\gamma$ has leads to antigen presentation during cell-mediated immunity accompanied by production of $\mathrm{T}$ helper (Th) 1 type pro-inflammatory cytokines, IL-1 $\beta$, IL-12 and tumor necrosis factor- $\alpha$. In contrast, the M2 polarization-induced by the Th2 type cytokines IL- 4 and IL-13 results in secretion of anti-inflammatory cytokines and expression of matrix metalloproteinase (MMP)-12 $[20,21]$. Although it is still uncertain which of these phenotypes is dominantly involved in COPD pathogenesis, there is compelling evidence that M1 polarized AM may play an important role in lung tissue destruction and impaired efferocytosis in response to cigarette smoke exposure $[22,23]$. Intriguingly, AIM has been demonstrated to be mainly expressed in M1 polarized macrophages in 


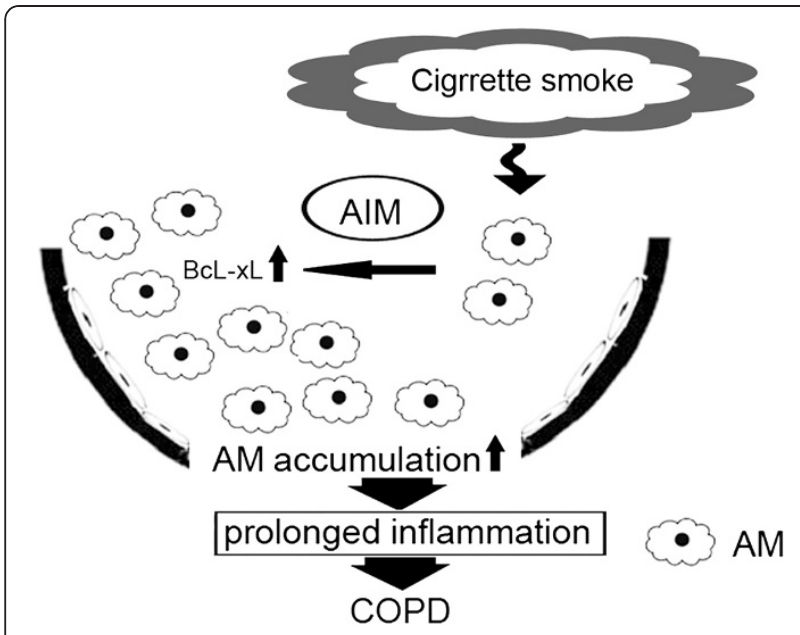

Figure 5 Hypothetical model of involvement of AIM in COPD pathogenesis. AIM expression AM in response to CSE exposure may enhance accumulation of AM as a pathogenic sequence for COPD development through prolonged inflammation. Definition of abbreviations: $\mathrm{AIM}=$ apoptosis inhibitor of macrophage, $\mathrm{AM}=$ alveolar macrophage, $\mathrm{BCl}-\mathrm{xL}=$ anti-apoptotic $\mathrm{B}$ cell lymphoma leukemia $\mathrm{COPD}=$ chronic obstructive pulmonary disease.

adipose tissue in obese mice [12]. Although the association between AM polarization and AIM expression in COPD is still unclear, it is plausible that the M1 polarized pro-inflammatory AM is mainly involved in AIM expression and chronicity of inflammation for COPD pathogenesis.

Although the detailed mechanism in in vitro CSE exposure remains to be determined, we speculate that oxLDL, a known strong inducer for AIM in atherosclerotic lesion, may be also involved in the mechanism for CSE-induced AIM expression in AM [13]. The presence of oxLDL in alveolar space of COPD lung has not been clearly demonstrated, however the experimental mouse model of lung edema induced by alpha-naphthylthiourea (ANTU) administration demonstrated existence of oxLDL in lung [24]. Indeed, oxLDL has been implicated in the pathogenesis for not only metabolic disorders but also several lung diseases such as asthma, acute lung injury, and cystic fibrosis through the surfactant regulation and inflammatory reactions $[13,25]$. Furthermore, oxLDL is one of the major and early lipid peroxidation products under the condition of increased oxidative stress, suggesting that cigarette smoke-induced highly oxidative microenvironment may enhance production of oxLDL in COPD lung. Among scavenger receptors, CD36 and macrophage scavenger receptor-1 (MSR1) are important for oxLDL uptake and AIM expression. One recent paper demonstrated that MSR1 expression is upregulated in AM of smokers [26]. In addition, polymorphisms in the MSR1 gene (MSR1) accompanied by increased MSR1 expression were associated with COPD susceptibility, lung function, and macrophage function [27]. Interestingly, MSR1 expression was also implicated in increase in cell number of monocyte-derived macrophage (MDM) in in vitro experiment [27]. Therefore, it is of particular interest to hypothesize that there is an association between MSR1 expression and up-regulation of AIM through the mechanisms of oxLDL uptake.

\section{Conclusions}

In summary, we have clarified the AIM expression in AM, which is enhanced in response to CSE exposure. The antiapoptotic property of AIM to CSE-induced apoptosis may at least partly account for the mechanism of prolonged survival and increase in number of $\mathrm{AM}$ as a pathogenic sequence for COPD development (Figure 5). The antiapoptotic property of AIM is against to multiple apoptosisinducing stimuli and also for multiple cell types including lymphocytes, and AIM was originally identified as an important regulator for fat metabolism [28]. Hence, future studies need to address the involvement of AIM in different aspects, including systemic inflammation and metabolic disorders in association with COPD pathophysiology [29].

\section{Competing interests}

None of the authors has a financial relationship with a commercial entity that has an interest in the subject of this manuscript.

\section{Authors' contributions}

JK, JA, HH, SI, NT, KK, SF, CT, TN, TI, KS, MK, KS, HH, SA, TM, YK, KN, and KK were involved in the conception and design of experiments, analyzed the data, and wrote the manuscript. JK, JA, HH, SI, and NT performed the experiments. JK, SI, NT, KK, JH, MO, and TM obtained informed consent and collected human samples. All authors read and approved the final manuscript.

\section{Acknowledgements}

We wish to thank Stephanie Cambier of the University of Washington for technical support. This work was supported by grants from the Jikei University Research fund to JK, JA, and KK. A Grant-In-Aid for Scientific Research from the Ministry of Education to JA, HH, CT, TN, KN, and KK.

\section{Author details}

'Department of Internal Medicine, Division of Respiratory Diseases, Jikei University School of Medicine, 3-25-8 Nishi-shimbashi, Minato-ku, Tokyo 105-8461, Japan. ${ }^{2}$ Department of Surgery, Division of Chest Diseases, Jikei University School of Medicine, Tokyo, Japan. ${ }^{3}$ Department of Pathology, Jikei University School of Medicine, Tokyo, Japan. ${ }^{4}$ Laboratory of Molecular Biomedicine for Pathogenesis, Center for Disease Biology and Integrative Medicine, Faculty of Medicine, The University of Tokyo, Tokyo, Japan.

Received: 7 January 2013 Accepted: 1 March 2013

Published: 5 March 2013

\section{References}

1. Eisner MD, Anthonisen N, Coultas D, Kuenzli N, Perez-Padilla R, Postma D, Romieu I, Silverman EK, Balmes JR: An official american thoracic society public policy statement: novel risk factors and the global burden of chronic obstructive pulmonary disease. Am J Respir Crit Care Med 2010, 182(5):693-718.

2. Barnes PJ: Alveolar macrophages as orchestrators of COPD. COPD 2004, 1:59-70.

3. Tetley TD: Macrophages and the pathogenesis of COPD. Chest 2002 121(5 Suppl):156S-159S.

4. Brusselle GG, Joos GF, Bracke KR: New insights into the immunology of chronic obstructive pulmonary disease. Lancet 2011, 378(9795):1015-1026. 
5. Barnes PJ: Immunology of asthma and chronic obstructive pulmonary disease. Nat Rev Immunol 2008, 8(3):183-192.

6. Di Stefano A, Capelli A, Lusuardi M, Balbo P, Vecchio C, Maestrelli P, Mapp CE, Fabbri LM, Donner CF, Saetta M: Severity of airflow limitation is associated with severity of airway inflammation in smokers. Am J Respir Crit Care Med 1998, 158:1277-1285.

7. Vlahos R, Bozinovski S, Chan SP, Ivanov S, Lindén A, Hamilton JA, Anderson GP: Neutralizing granulocyte/macrophage colony-stimulating factor inhibits cigarette smoke-induced lung inflammation. Am J Respir Crit Care Med 2010, 182:34-40.

8. Kuwano K, Araya J, Nakayama K: Epithelial cell fate following lung injury. Expert Rev Respir Med 2008, 2(5):573-582.

9. Kasahara Y, Tuder RM, Taraseviciene-Stewart L, Le Cras TD, Abman S, Hirth PK, Waltenberger J, Voelkel NF: Inhibition of VEGF receptors causes lung cell apoptosis and emphysema. J Clin Invest 2000, 106(11):1311-1319.

10. Siganaki M, Koutsopoulos AV, Neofytou E, Vlachaki E, Psarrou M, Soulitzis N Pentilas N, Schiza S, Siafakas NM, Tzortzaki EG: Deregulation of apoptosis mediators' p53 and bcl2 in lung tissue of copd patients. Respir Res 2010, 11:46.

11. Tomita K, Caramori G, Lim S, Ito K, Hanazawa T, Oates T, Chiselita I, Jazrawi E, Chung KF, Barnes PJ, Adcock IM: Increased p21(Cip1/Waf1) and B cell lymphoma leukemia-x(L) expression and reduced apoptosis in alveolar macrophages from smokers. Am J Respir Crit Care Med 2002, 166(5):724-731.

12. Miyazaki T, Hirokami Y, Matsuhashi N, Takatsuka H, Naito M: Increased susceptibility of thymocytes to apoptosis in mice lacking AIM, a novel murine macrophage-derived soluble factor belonging to the scavenger receptor cysteine-rich domain superfamily. J Exp Med 1999, 189(2):413-422.

13. Arai S, Shelton JM, Chen M, Bradley MN, Castrillo A, Bookout AL, Mak PA, Edwards PA, Mangelsdorf DJ, Tontonoz P, Miyazaki T: A role for the apoptosis inhibitory factor Aim/Spalpha/Api6 in atherosclerosis development. Cell Metab 2005, 1(3):201-213.

14. Kuwata K, Watanabe H, Jiang SY, Yamamoto T, Tomiyama-Miyaji C, Abo T, Miyazaki T, Naito M: Aim inhibits apoptosis of T cells and NKT cells in corynebacterium-induced granuloma formation in mice. Am J Pathol 2003, 162(3):837-847.

15. Araya J, Cambier S, Markovics JA, Wolters $P$, Jablons D, Hill A, Finkbeiner W, Jones K, Broaddus VC, Sheppard D, Barzcak A, Xiao Y, Erle DJ, Nishimura SL: Squamous metaplasia amplifies pathologic epithelial-mesenchymal interactions in COPD patients. J Clin Invest 2007, 117(11):3551-3562.

16. Araya J, Maruyama M, Inoue A, Fujita T, Kawahara J, Sassa K, Hayashi R, Kawagishi Y, Yamashita N, Sugiyama E, Kobayashi M: Inhibition of proteasome activity is involved in cobalt-induced apoptosis of human alveolar macrophages. Am J Physiol Lung Cell Mol Physiol 2002, 283(4): L849-858.

17. Fujii S, Hara H, Araya J, Takasaka N, Kojima J, Ito S, Minagawa S, Yumino Y, Ishikawa T, Numata T, Kawaishi M, Hirano J, Odaka M, Morikawa T, Nishimura S, Nakayama K, Kuwano K: Insufficient autophagy promotes bronchial epithelial cell senescence in chronic obstructive pulmonary disease. Oncolmmunology 2012, 1(5):630-641.

18. Numata T, Araya J, Fujii S, Hara H, Takasaka N, Kojima J, Minagawa S, Yumino Y, Kawaishi M, Hirano J, Odaka M, Morikawa T, Nishimura SL, Nakayama K, Kuwano K: Insulin-dependent phosphatidylinositol 3-kinase /Akt and ERK signaling pathways inhibit TLR3-mediated human bronchial epithelial cell apoptosis. J Immunol 2011, 187(1):510-519.

19. Hu W, Xie J, Zhao J, Xu Y, Yang S, Ni W: Involvement of Bcl-2 family in apoptosis and signal pathways induced by cigarette smoke extract in the human airway smooth muscle cells. DNA Cell Biol 2009, 28(1):13-22.

20. Shaykhiev R, Krause A, Salit J, Strulovici-Barel Y, Harvey BG, O'Connor TP Crystal RG: Smoking-dependent reprogramming of alveolar macrophage polarization: implication for pathogenesis of chronic obstructive pulmonary disease. J Immunol 2009, 183(4):2867-2883.

21. Byers DE, Holtzman MJ: Alternatively activated macrophages and airway disease. Chest 2011, 140(3):768-774.

22. Barnes PJ: The cytokine network in chronic obstructive pulmonary disease. Am J Respir Cell Mol Biol 2009, 41(6):631-638.

23. Hodge S, Matthews G, Mukaro V, Ahern J, Shivam A, Hodge G, Holmes M, Jersmann H, Reynolds PN: Cigarette smoke-induced changes to alveolar macrophage phenotype and function are improved by treatment with procysteine. Am J Respir Cell Mol Biol 2011, 44(5):673-681.

24. Sipahi EY, Ozel Tekin I, Comert M, Barut F, Ustun H, Sipahi TH: Oxidized low-density lipoproteins accumulate in rat lung after experimental lung edema induced by alpha- naphthylthiourea (ANTU). Pharmacol Res 2004, 50(6):585-591.

25. Zhou J, Ryan AJ, Medh J, Mallampalli RK: Oxidized lipoproteins inhibit surfactant phosphatidylcholine synthesis via calpain-mediated cleavage of CTP: Phosphocholine cytidylyltransferase. J Biol Chem 2003, 278(39): 37032-37040.

26. Heguy A, O'Connor TP, Luettich K, Worgall S, Cieciuch A, Harvey BG, Hackett NR, Crystal RG: Gene expression profiling of human alveolar macrophages of phenotypically normal smokers and nonsmokers reveals a previously unrecognized subset of genes modulated by cigarette smoking. J Mol Med (Berl) 2006, 84(4):318-328.

27. Ohar JA, Hamilton RF Jr, Zheng S, Sadeghnejad A, Sterling DA, Xu J, Meyers DA, Bleecker ER, Holian A: COPD is associated with a macrophage scavenger receptor-1 gene sequence variation. Chest 2010, 137(5):1098-1107.

28. Kurokawa J, Arai S, Nakashima K, Nagano H, Nishijima A, Miyata K, Ose R, Mori M, Kubota N, Kadowaki T, Oike Y, Koga H, Febbraio M, Iwanaga T, Miyazaki T: Macrophage-derived AIM is endocytosed into adipocytes and decreases lipid droplets via inhibition of fatty acid synthase activity. Cell Metab 2010, 11(6):479-492.

29. van den Borst B, Gosker HR, Wesseling G, de Jager W, Hellwig VA, Snepvangers FJ, Schols AM: Low-grade adipose tissue inflammation in patients with mild-to-moderate chronic obstructive pulmonary disease. Am J Clin Nutr 2011, 94(6):1504-1512.

doi:10.1186/1465-9921-14-30

Cite this article as: Kojima et al:: Apoptosis inhibitor of macrophage (AIM) expression in alveolar macrophages in COPD. Respiratory Research 2013 14:30.

\section{Submit your next manuscript to BioMed Central and take full advantage of:}

- Convenient online submission

- Thorough peer review

- No space constraints or color figure charges

- Immediate publication on acceptance

- Inclusion in PubMed, CAS, Scopus and Google Scholar

- Research which is freely available for redistribution 